ISSN 0103-9954

\title{
CRESCIMENTO INICIAL E QUALIDADE DE MUDAS DE Pterogyne nitens Tull. CONDUZIDAS SOB DIFERENTES NÍVEIS DE RESTRIÇÃO LUMINOSA ARTIFICIAL
}

\author{
INITIAL GROWTHAND QUALITY OF Pterogyne nitens Tull.SEEDLING UNDER ARTIFICIAL \\ SHADING GRADIENT
}

\author{
Fábio Ricardo Coutinho Fontes César ${ }^{1}$ Sylvana Naomi Matsumoto ${ }^{2}$ Anselmo Eloy Silveira Viana ${ }^{3}$ \\ Joice Andrade Bonfim ${ }^{4}$
}

\section{RESUMO}

Objetivou-se com este estudo avaliar o comportamento de plantas de Pterogyne nitens Tull. sob diferentes níveis de restrição luminosa durante seu desenvolvimento inicial. O estudo foi conduzido no período de março a junho de 2008, no campo agropecuário da Universidade Estadual do Sudoeste da Bahia - UESB, em Vitória da Conquista, BA. Sete experimentos foram definidos por níveis de 20\%, 30\%, 40\%, 50\%, 60\% e $70 \%$ de restrição luminosa e plantas mantidas a pleno sol. Cada um dos experimentos foi organizado em delineamento experimental inteiramente casualizado, com cinco repetições e parcelas constituídas por 12 plantas. Durante o período de 15 a 75 dias após a emergência das plântulas, em intervalos de 15 dias, foram avaliadas as características morfológicas e quantitativas de crescimento e o índice de qualidade Dickson (IQD). Os gradientes de restrição de luz induziram a alterações morfológicas como elevação da altura e aumento da área foliar individual e total das plantas. Reduções de massa do sistema radicular ocorreram em detrimento de tais modificações. Invariavelmente, os maiores índices de IQD foram verificados para as plantas mantidas sob 36,5\% de restrição luminosa.

Palavras-chave: viveiro; sombreamento; luz; índice de qualidade.

\begin{abstract}
The objective of this study was to evaluate the initial development and quality of seedlings Pterogyne nitens Tull., produced under different luminosity levels. The experiment was conducted from March to June 2008, in the agricultural field at the State University of Southwestern Bahia state - UESB, in Vitoria da Conquista, BA state. Seven experiments were defined for levels of $20 \%, 30 \%, 40 \%, 50 \%, 60 \%$ and $70 \%$ of light restriction and plants kept in full sun. For each experiment was followed the completely randomized design with five replications and each experimental unit was composed for 12 plants. In a period from 15 to 75 days after the plant emergence, at intervals of 15 days, the morphological and quantitative characteristics of growth and quality index of Dickson (IQD) were evaluated. The constraint of light gradients induced morphological changes such as raising the height and increased individual and total leaf area of plants. Reductions in root system mass occurred at the expense of such modifications. Invariably, the highest rates were observed for the IQD plants lied under 36.5\% light restriction.
\end{abstract}

Keywords: nursery; shading; light; quality index.

1 Engenheiro Agrônomo, Msc., Doutorando em Solos e Nutrição de Plantas, Universidade de São Paulo, Escola Superior de Agricultura Luiz de Queiroz, Av. Pádua Dias, 11, Caixa Postal 9, CEP 13418-900, Piracicaba (SP), Brasil.fabiorc.agro@gmail.com

2 Engenheira Agrônoma, Dra., Professora Titular do Departamento de Fitotecnia e Zootecnia, Laboratório de Fisiologia Vegetal, Universidade Estadual do Sudoeste da Bahia. Estrada do Bem Querer, Km 4, CEP 45083-900, Vitoria da Conquista (BA), Brasil.sylvananaomi@yahoo.com.br

3 Engenheiro Agrônomo, Dr.,Professor Titular do Departamento de Fitotecnia e Zootecnia, Laboratório de Produção e Melhoramento Vegetal, Universidade Estadual do Sudoeste da Bahia. Estrada do Bem Querer, Km 4, CEP 45.083-900, Vitoria da Conquista (BA), Brasil.ae-viana@uol.com.br

4 Engenheira Agrônoma, Msc., Doutoranda em Solos e Nutrição de Plantas, Universidade de São Paulo, Escola Superior de Agricultura Luiz de Queiroz, Av. Pádua Dias, 11, Caixa Postal 9, CEP 13418-900, Piracicaba (SP), Brasil. joice.agro@yahoo.com.br

Recebido para publicação em 8/11/2011 e aceito em 8/10/2012 


\section{INTRODUÇÃO}

Pterogyne nitens Tull. (família Fabaceae, subfamília Caesalpinioideae) é uma espécie arbórea, conhecida como "madeira nova" e "amendoim-do-campo", sendo caracterizada por sua rusticidade e elevadas taxas de crescimento (FILARDI et al., 2007, LORENZI, 2002, PELLIZZARO et al., 2011). Tem ocorrência descrita na Argentina, Bolívia, Paraguai e Brasil; no Brasil ocorre na região Nordeste estendendo-se até o Oeste de Santa Catarina, em ambientes da caatinga, cerrado e formações florestais, pluvial e estacional (FILARDI et al., 2009, REGAZINI et al., 2008). É uma árvore cuja utilização é recomendada para arborização de vias urbanas e rodovias, reposição de mata ciliar para locais com inundações periódicas e revegetação em sítios arenosos e degradados (LORENZI, 2002). Sua madeira é bastante utilizada na construção de móveis finos e é empregada na construção civil, devido a sua alta densidade $\left(0,77 \mathrm{~g} \mathrm{~cm}^{-2}\right)$ e resistência moderada (LORENZI, 2002). De acordo com Ferreira et al. (2009), há indícios de que esta espécie esteja sob riscos de extinção fazendo parte da lista de espécies arbóreas recomendadas para conservação genética no Estado de São Paulo.

Apesar da importância econômica e ambiental, até o presente momento, a base de conhecimentos construída para esta espécie é insuficiente para a definição de práticas de manejo que permitam o pleno desenvolvimento de estratégias para conservação e para a constituição de uma cadeia produtiva. A adequação de formas de propagação e estrutura da árvore são aspectos que limitam tal processo. É de fundamental importância a obtenção de mudas com elevada taxa de sobrevivência e crescimento após o plantio no campo, fato que condiciona o sucesso de implantação de qualquer espécie no contexto de um sistema florestal, qualquer que seja a natureza de sua finalidade. Para fins de manejo, um fato a ser considerado é a reduzida altura do fuste principal e estrutura da copa com intensa ramificação, ocasionados pela precocidade da perda da dominância apical. Alternativas de manejo da disponibilidade de luz por meio da elevação da densidade de plantas por área e a produção de mudas sob restrição de luz para indução de maior altura e manutenção da dominância apical podem ser propostas como formas estratégicas de redução de tal entrave.

A capacidade de adaptação às condições de luminosidade do ambiente é o principal fator considerado na classificação ecológica das árvores. A espécie Pterogyne nitens é classificada como perenifólia, semicaducifolia e heliófita; na escala sucessional é definida como uma espécie secundária inicial, ocorrendo em capoeiras, podendo comportar-se como pioneira em locais arenosos e degradados (CARVALHO, 1994). Entretanto para a classificação quanto à sucessão ecológica, outros aspectos da interação entre as plantas e fatores intrínsecos ao habitat e ao nicho ecológico da espécie além da disponibilidade de radiação devem ser considerados.

De acordo com Ruberti et al. (2011), existem dois mecanismos de resposta das plantas à restrição de luz: tolerância e escape. As plantas que apresentam tolerância ao sombreamento são caracterizadas por condicionar adaptações da fotossíntese a situações de baixa luminosidade, sendo capazes de sobreviver durante um longo período de tempo sob um dossel sombreado. Plantas que possuem o mecanismo de escape ao sombreamento adaptam seu crescimento para maximizar a intercepção de luz e, desta forma, rapidamente, ocupar as lacunas do dossel.

Variações nos níveis de intensidade luminosa podem afetar alguns aspectos morfofisiológicos das plantas como elevação da altura, redução do diâmetro do caule, redução do número de internódios, maior expansão da área foliar individual, teores de clorofila mais altos quando relacionados à área foliar, sendo o conjunto de alterações ontogênicas denominados de "síndrome da evitação ao sombreamento". Entretanto, embora seja clara a importância biológica deste fenômeno, quando as árvores nativas são submetidas ao manejo cultural, tais alterações devem ser direcionadas para que propiciem a inserção de espécies nativas ao contexto produtivo. De acordo com Ruberti et al. (2011), o maior desafio é, portanto, expandir o conhecimento sobre mecanismos de resposta e adaptação a alterações na qualidade da luz incidente nas plantas como um todo e em órgãos individualizados para que sejam delineadas novas estratégias, visando à produção das culturas sob elevada densidade de plantio.

Objetivou-se com este estudo avaliar como o gradiente de restrição artificial de radiação solar pode afetar o desenvolvimento inicial e a qualidade de mudas de Pterogyne nitens Tull.

\section{MATERIAL E MÉTODOS}

O experimento foi conduzido no período 
de março a junho de 2008, no campo agropecuário experimental da Universidade Estadual do Sudoeste da Bahia - UESB, em Vitória da Conquista, BA, situado a $14^{\circ} 53^{\prime}$ ' latitude Sul e $40^{\circ} 48^{\prime}$ longitude Oeste, a altitude de $870 \mathrm{~m}$, com temperatura média anual de $20,2^{\circ} \mathrm{C}$ e precipitação anual de $900 \mathrm{~mm}$.

$\mathrm{O}$ estudo foi composto por sete experimentos constituídos por diferentes níveis de restrição de luz $(20,30,40,50,60$ e $70 \%)$ e a pleno sol. As condições de restrição de luz foram obtidas em diferentes viveiros com dimensões de $6,0 \times 9,0 \times 2,20 \mathrm{~m}$, totalmente cobertos com sombrites de malha preta com especificações de $20,30,40,50,60$ e $70 \%$ de restrição luminosa. Os viveiros foram construídos no sentido perpendicular ao eixo leste-oeste de percurso diário do sol, evitando a sobreposição de sombra entre os telados.

Para formação das mudas foram utilizados sacos de polietileno de dimensões 19 × $36 \mathrm{~cm}$ e capacidade para $4 \mathrm{~kg}$ de substrato, que foi composto por subsolo retirado da camada abaixo de $20 \mathrm{~cm}$ mais esterco de curral curtido na proporção $3: 1$, acrescido de adubação básica com $25 \mathrm{mg} \mathrm{kg}^{-1}$ de $\mathrm{P}$ (superfosfato simples) e $6 \mathrm{mg} \mathrm{kg}^{-1} \mathrm{de} \mathrm{K}$ (cloreto de potássio). Após o preparo dos recipientes, estes foram dispostos nos viveiros em espaçamento de $30 \times 30 \mathrm{~cm}$.

Foram semeadas três sementes por recipiente, coletadas de aproximadamente 30 árvores matrizes localizadas no Campus da Universidade Estadual do Sudoeste da Bahia (UESB). As vagens completamente maduras, contendo as sementes, foram colhidas no chão, sob as árvores. Para a quebra de dormência foi utilizado tratamento com imersão em água a $60^{\circ} \mathrm{C}$ em banho-maria, em períodos de 5 minutos (MARTINS NETO et al., 2009). Aos cinco dias após a emergência foi feito o desbaste das plântulas deixando somente uma por recipiente. Durante a condução do experimento foi feito o controle das plantas invasoras manualmente e as regas foram realizadas diariamente para manter o substrato em condição de capacidade de campo.

Para cada um dos experimentos foi seguido o delineamento experimental inteiramente casualizado, com cinco repetições e parcelas constituídas por 12 plantas. Os paramentos de crescimento foram avaliados a cada 15 dias, no período de 15 a 75 dias após a emergência das plantas, totalizando 5 avaliações, sendo essas definidas como os tratamentos de cada experimento.

Para a determinação da altura das plantas $(\mathrm{H})$, foi medida a distância entre o colo da muda até o ápice, expressa (cm); o diâmetro do colo (D) foi determinado com auxílio de um paquímetro, expresso em $(\mathrm{mm})$. Determinou-se à área foliar total (AF), expresso em $\mathrm{cm}^{2}$, utilizando o medidor de área foliar LI-3100 Área Meter, LI-COR, USA; o número de folhas (NF) foi determinado por meio da contagem direta.

Em cada coleta foram retiradas duas mudas por parcela para avaliações destrutivas de massa seca. Após a amostragem, as plantas foram acondicionadas em sacos plásticos identificados e imediatamente divididas em parte aérea e sistema radicular. As raízes foram lavadas para eliminar o substrato aderido. Após tal procedimento o material foi submetido à estufa com circulação de ar forçado a $65^{\circ} \mathrm{C}$ por 48 horas. Em seguida procedeu-se a pesagem em balança analítica, para determinação da massa seca expressa em (g).

Foram avaliadas as relações entre os paramentos de altura das plantas e diâmetro do colo (H/D) e massa seca da parte aérea com massa seca da raiz (MSPA/MSR), e o índice de qualidade Dickson (IQD) calculado de acordo com Dickson et al. (1960)

$$
\mathrm{IQD}=\frac{\mathrm{MST}(\mathrm{g})}{\frac{\mathrm{H}(\mathrm{cm})}{\mathrm{D}(\mathrm{mm})}+\frac{\mathrm{MSPA}(\mathrm{g})}{\operatorname{MSR}(\mathrm{g})}}
$$

Os dados foram submetidos à análise de variância individual para cada experimento, sendo realizada a análise conjunta dos experimentos quando a relação entre os quadrados médios do resíduo foi menor ou igual 1:6. O estudo dos níveis de restrição luminosa (RL) e dos dias após a emergência das plantas (DAE) (avaliações) foi feito através da análise de variância e empregando-se a metodologia de superfície de resposta utilizando o pacote estatístico "Statistical Analysis System" SAS (SAS INSTITUTE, 1985), sendo os modelos definidos de acordo com comportamento biológico e o coeficiente de determinação.

\section{RESULTADOS E DISCUSSÃO}

Foi verificada interação entre os níveis de restrição de luz (RL) e dias após a emergência (DAE) para todas as variáveis dependentes estudadas, sendo realizado o desdobramento segundo a metodologia da superfície de resposta (Tabela 1). Para todos os parâmetros de crescimento avaliados foi verificada tendência de acréscimos de valores com o decorrer de DAE. 
TABELA 1: Quadrados médios e graus de liberdade (GL) referente à análise de variância da altura (H), diâmetro do solo (D), número de folhas (NF), área foliar (AF), massa seca da raiz (MSR), massa seca da parte aérea (MSPA), massa seca total (MST), da relação entre altura e diâmetro do colo (H/D), massa seca da parte aérea com massa seca da raiz (MSPA/MSR) e o índice de qualidade de Dickson (IQD) em função do número de dias após a emergência (DAE) e níveis de restrição luminosa $(\mathrm{RL})$.

TABLE 1: Mean squares and degrees of freedom (GL) on the analysis of variance of height (H), collar diameter (D), number of leaves (NF), leaf area (AF), root dry weight (MSR), aerial part dry weight (MSPA), total dry weight (MST), and the relationship between height of the aerial part per collar diameter (H/DC) and the aerial par with dry weight per dry weight root (MSPA/ MSR) and quality index of Dickson (IQD) in function to number of days after emergence (DAE) and luminosity levels (RL).

\begin{tabular}{|c|c|c|c|c|c|c|c|c|c|c|c|}
\hline \multirow{2}{*}{$\begin{array}{l}\text { Causa de } \\
\text { Variação }\end{array}$} & \multirow[b]{2}{*}{ GL } & \multicolumn{10}{|c|}{ Quadrados Médios } \\
\hline & & $\mathrm{H}$ & $\mathrm{D}$ & NF & $\mathrm{AF}$ & MSR & MSPA & MST & $\mathrm{H} / \mathrm{D}$ & $\begin{array}{l}\text { MSPA/ } \\
\text { MSR }\end{array}$ & IQD \\
\hline $\mathrm{RL}$ & 6 & $470,9^{* *}$ & $6,7^{\mathrm{ns}}$ & $5,1^{* *}$ & $268145,7^{* *}$ & $1,84^{*}$ & $10,3^{* *}$ & $219,2^{* *}$ & $16,0^{* *}$ & $5,4^{\mathrm{ns}}$ & $0,4^{*}$ \\
\hline $\mathrm{D}$ & 4 & $2074,8^{* *}$ & $50,4^{* *}$ & $377,2^{* *}$ & $2007638,0^{* *}$ & $40,1^{* *}$ & $203,9^{* *}$ & $845,6^{* *}$ & $21,5^{* *}$ & $51,4^{* *}$ & $7,8^{* *}$ \\
\hline$R L \times D$ & 24 & $45,40^{* *}$ & $3,53^{* *}$ & $0,96^{* *}$ & $31140,29^{* *}$ & $0,73^{* *}$ & $1,68^{* *}$ & $31,25^{* *}$ & $1,23^{* *}$ & $2,55^{* *}$ & $0,15^{* *}$ \\
\hline Resíduo & 140 & 4,74 & 0,04 & 0,39 & 3566,75 & 0,13 & 0,45 & 1,01 & 0,29 & 0,95 & 0,02 \\
\hline Total & 174 & & & & & & & & & & \\
\hline
\end{tabular}

Valores máximos de altura $(36,5 \mathrm{~cm})$ e área foliar $\left(874,22 \mathrm{~cm}^{2}\right)$ foram observados para a maior restrição de radiação $(70 \%)$ ao final do experimento (75 dias) (Figuras 1a e 1b). Durante todo o período do estudo foi delineado um comportamento linear crescente para altura e quadrático para a área foliar em função da intensidade de restrição de luz e do tempo. A restrição da radiação induz ao estiolamento da planta, sendo esse mecanismo ocasionado pela interação de hormônios vegetais (VEGLIO, 2010). Segundo o mesmo autor, sob restrição de luz, os níveis de auxina biossintetizada pela planta são modulados por dois fatores: o ambiente e a ontogenia da planta. $\mathrm{O}$ fator ambiental está relacionado ao decréscimo da relação entre luz vermelha e luz vermelha distante (TAO et al., 2008). $O$ fator ontogenético seria mediado pela síntese de etileno que estimula a produção de auxinas mesmo sem a presença de plantas vizinhas (STEPANOVA, 2008). De acordo com Franco et al. (2007), independentemente da tolerância das plantas, o incremento da altura, seja este ocorrente no início ou em estágios posteriores de desenvolvimento, seria uma resposta favorável à sobrevivência da planta sob condição de sombreamento, em virtude de um crescimento líquido global positivo considerado como fator de maior importância (LUSK, 2004).

Para o período entre 15 a 33 dias, a maximização de AF foi favorecida por níveis intermediários de sombreamento, cujos valores limites foram sucessivamente elevados com o decorrer do tempo. Do $34^{\circ}$ dia até o final do experimento, foi mantida uma relação estritamente de acréscimos de $\mathrm{AF}$ em relação à elevação de restrição luminosa. Apesar da alteração de comportamento de $\mathrm{AF}$ em relação à restrição luminosa, quando a AF individual foi analisada, foi verificado efeito homogêneo de RL durante todo o período do ensaio, sendo observados valores $47 \%$ maiores de acréscimo da AF individual para a condição de 70\% de RL quando comparada ao tratamento a pleno sol $\left(26,59 \mathrm{~cm}^{2}\right.$ e $57,05 \mathrm{~cm}^{2}$ de acréscimo de área para folhas individuais para a condição a pleno sol e $70 \%$ de RL, respectivamente, durante o período de 15 a 75 DAE). O estiolamento e a expansão da área foliar são as alterações morfológicas mais comuns em situações de restrição luminosa, sendo caracterizados como mecanismos estratégicos para otimizar a eficiência de interceptação da radiação (MORAES NETO et al., 2000). Esse comportamento 
a)

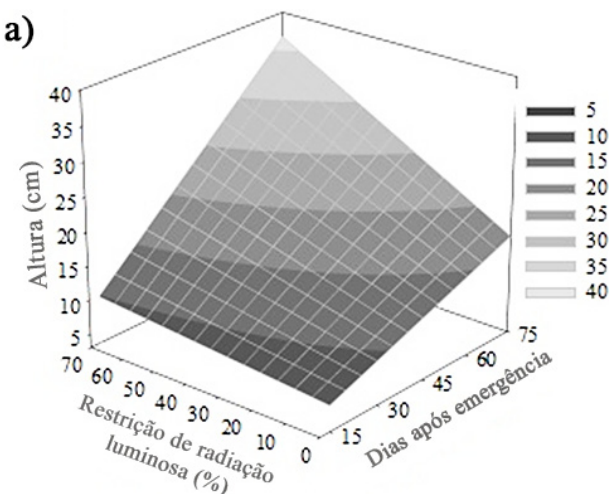

$\hat{y}^{1}=4,16561-0,01303^{\text {ns }} R+0,17431^{1} \mathrm{D}+0,00383^{1} R D$ $\left(\mathrm{R}^{2}=0,85\right)$

c)

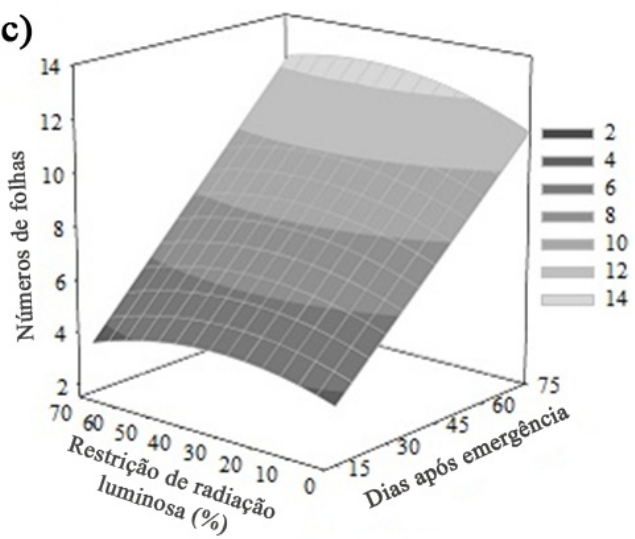

$\hat{\mathbf{y}}^{1}=1,76465+0,04042^{1} \mathrm{R}+0,12539^{1} \mathrm{D}-0,00068^{1} \mathrm{R}^{2}+$ $0,00034^{1} \mathrm{RD}\left(\mathrm{R}^{2}=0,95\right)$ b)

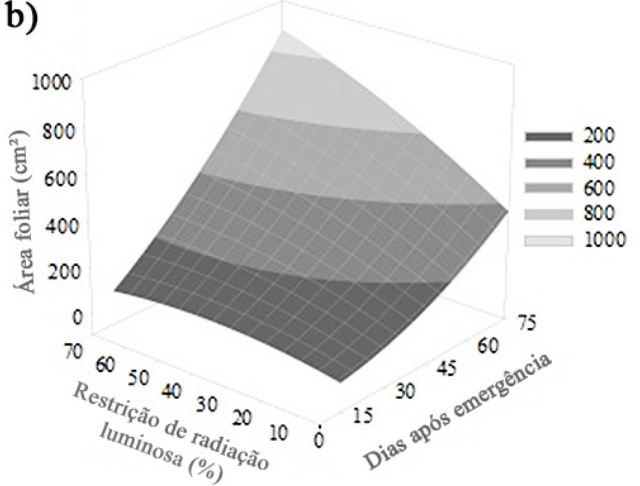

$\hat{y}^{1}=6,36962+1,47779^{\text {ns }} \mathrm{R}-0,55340^{\text {ns }} \mathrm{D}-0,03947^{1} R^{2}+$ $0,11947^{1} \mathrm{RD}+0,06609^{1} \mathrm{D}^{2}\left(\mathrm{R}^{2}=0,92\right)$

d)

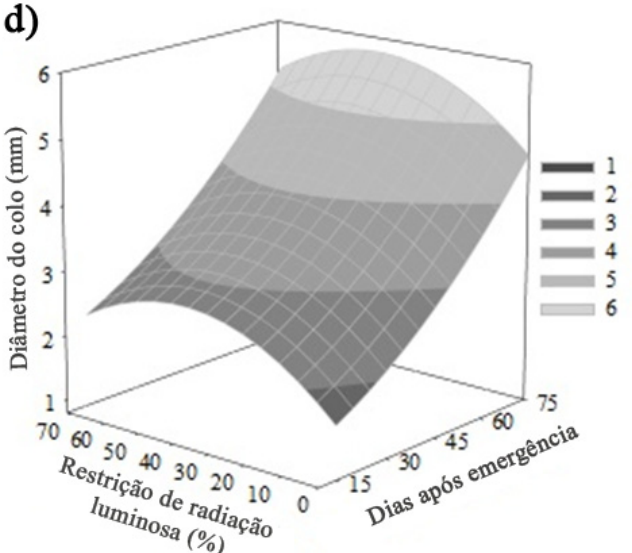

$\hat{\mathrm{y}}^{1}=1,26832+0,05571^{1} \mathrm{R}+0,01721^{\text {ns }} \mathrm{D}-0,00066^{1} \mathrm{R}^{2}+$ $0,00036^{1} \mathrm{D}^{2}\left(\mathrm{R}^{2}=0,66\right)$

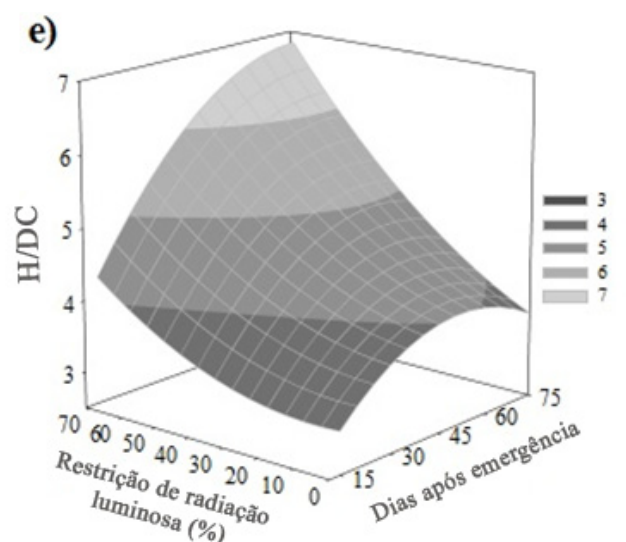

$\hat{y}^{1}=2,16487-0,01103^{\text {ns }} R+0,07177^{1} D+0,00030^{1} R^{2}+0,00047^{1} R D-0,00069^{1} D^{2}\left(R^{2}=0,69\right)$

FIGURA 1: Superfície de resposta para o crescimento em altura das plantas (a), área foliar (b), número de folhas (c), diâmetro do colo (d) e para a relação altura (H)/diâmetro do colo (D) (e) de Pterogyme nitens Tull., em função dos níveis de restrição de radiação luminosa (RL) e dias após a emergência (DAE) em Vitória da Conquista, Bahia. ${ }^{\text {ns }},{ }_{1}$ coeficientes da equação não significativo e significativo a 5 e $1 \%$, respectivamente.

FIGURE 1: Response surface of Pterogyne nitens Tull. height (a), leaf area (b), number of leaves (c), stem diameter growth (d), and height (H)/collar diameter (D) (e) growing at radiance restriction levels (R) and days after emergence (DAE) in Vitória da Conquista, Bahia. ${ }^{\text {ns }},{ }^{1}$ coefficients of the equation not significant and significant at the 5 and $1 \%$ respectively. 
tem sido verificado por vários autores e em diferentes espécies, sem qualquer relação com a necessidade de luz e sucessão populacional, Alvarenga et al. (2003) para Croton urucurana, Chaves e Paiva (2004) para Senna macranthera, Melo et al. (2008) para Enterolobium contortisiliquum e Caron et al. (2010) para Schizolobium parahyba.

Para o NF foi delineada resposta quadrática da RL e linear para DAE (Figura 1c), sendo verificado comportamento crescente em função do número de dias, com valor máximo de 12,66 verificado para $46,82 \%$ de RL, aos 75 dias. O maior NF observado sob condição de sombreamento intermediário durante todo o período de estudo pode estar relacionada à otimização da fotossíntese e das condições microclimáticas resultando em maior acúmulo de carboidratos e aceleração da emissão de folhas. Cesar et al. (2010) trabalhando com mudas de cafeeiros sob níveis de restrição luminosa de 0, 30, 50 e 70\%, também verificaram maior número de folhas para sombreamento intermediário $(\sim 30 \%)$.

A relação entre diâmetro do colo e restrição luminosa foi homogênea e definida pelo modelo quadrático, atingindo valores máximos estimados para o nível $42 \%$ de RL durante todo o período do ensaio (Figura 1d). De acordo com Valladares et al. (2011), há um maior investimento das plantas tolerantes ao sombreamento no aumento do diâmetro do colo em relação às plantas intolerantes. Azevedo et al. (2010) verificaram comportamento semelhante para mudas de Simarouba amara, sendo observados maiores valores de $\mathrm{D}$ na condição de $50 \%$ de RL.

Para a condição a pleno sol, aos 53 dias após emergência das plantas, foi alcançado o valor máximo da relação H/D $(4,03)$, sendo verificado decréscimo para as datas posteriores até o final do estudo (Figura 1e). Com a incidência do sombreamento, H/D foi elevada, atingindo os valores máximos em períodos mais tardios (6,83 para $70 \%$ de RL aos 75 DAE). Este comportamento devese ao estiolamento da planta simultaneamente com a redução do diâmetro do colo, resultando em aumento da relação H/D. Anten et al. (2009) verificaram que sob condição de restrição de luz, a ocorrência simultânea da redução do diâmetro do caule e alongamento dos internódios reduz a habilidade de autossustentação das plantas, elevando a susceptibilidade ao tombamento. Assim, relações H/D elevadas não são desejáveis, pois, podem estar relacionadas a um crescimento desordenado da parte aérea da planta.
Durante todo o período de avaliação, foi definido o modelo quadrático para a relação entre as características de massa (MSPA, MSR e MST) e RL. Inicialmente, aos 15 dias, foram verificados valores máximos de MSPA, MSR e MST sob condição muito semelhante de RL, próximas a 35\% (37\% para MSPA, $34 \%$ para MSR e $36 \%$ para MST). Foram estimados valores máximos aos 75 dias, para os níveis de RL $60 \%, 45 \%$ e $55 \%$, respectivamente para MSPA $(7,34 \mathrm{~g}), \operatorname{MSR}(2,93 \mathrm{~g})$ e MST $(10,09 \mathrm{~g})$ (Figuras 2a, 2b e 2c). Deste modo, foi verificado que, quando as plantas são submetidas à condição de exposição à radiação solar intensa, ocorreram menores valores de acúmulo de massa. Entretanto, quando a condição de RL foi elevada além de um ponto ótimo, houve decréscimos de massa. De acordo com Pooter e Kitajima (2007), plantas sob restrição luminosa tendem a direcionar seu metabolismo para a síntese de carboidratos não estruturais, visando a maior capacidade de sobrevivência, resultando em redução de acúmulo de massa.

Devido ao maior desvio de valores máximos ter ocorrido para a relação MSPA/MSR entre 15 e 75 dias $(37 \%$ de RL aos 15 DAE para $60 \%$ de RL aos $70 \mathrm{DAE}$ ), foi concluído que a modulação entre os níveis de restrição de luz e o estágio de desenvolvimento para esta característica foi mais intensa quando comparada aos efeitos de RL em relação à MSR, cuja variação foi menor, mantendo-se mais estável durante todo o período do ensaio (34\% aos 15 DAE comparados a $45 \%$ aos 75 DAE). Deste modo, foi observado que o custo das alterações morfológicas e metabólicas restringiu a capacidade de acúmulo de carboidratos estruturais sob menor taxa de sombreamento na fase inicial de desenvolvimento desta espécie. Ao final do ensaio, tal restrição foi verificada para níveis de sombreamento mais intenso. De acordo com Niinemets (2010), independentemente da condição ambiental, a redução das taxas de crescimento e aumento da proporção de carboidratos não estruturais ocorrem em função do decorrer dos estágios de desenvolvimento da espécie.

A redução de acúmulo da MSR pode estar relacionada à diminuição do fluxo descendente dos assimilados dos órgãos fotossintéticos à medida que a RL foi elevada, pois a luz é um dos fatores determinantes para esse transporte direcional (RESENDE et al., 2011). É importante ressaltar que mudas com um sistema radicular bem desenvolvido têm maior capacidade de sobrevivência no campo, conforme relatado por Campos e Uchida 

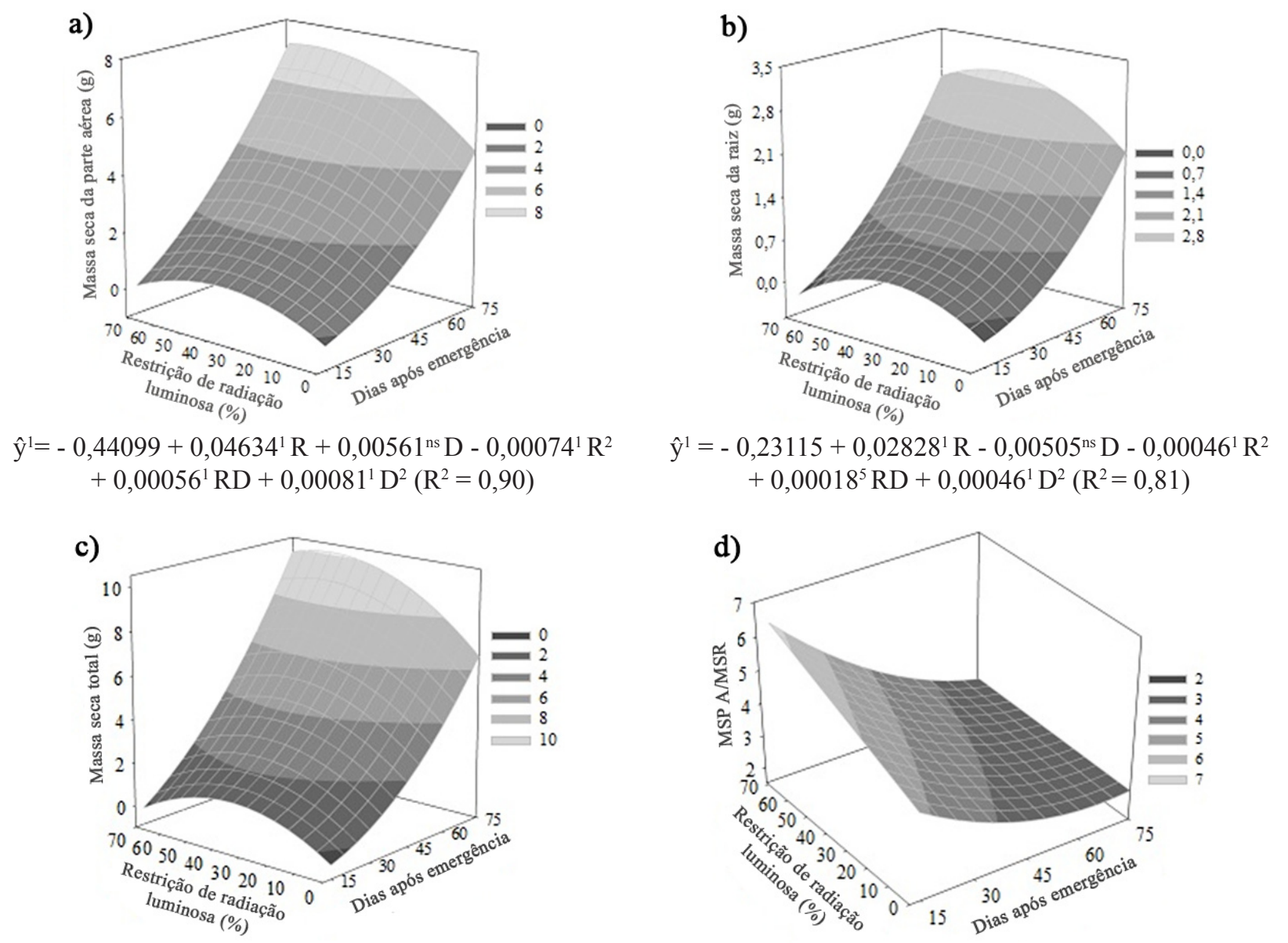

$\hat{y}^{1}=-0,67821+0,07469^{1} R+0,00084^{\text {ns }} D-0,00119^{1} R^{2}$
$+0,00074^{1} R D+0,00127^{1} D^{2}\left(R^{2}=0,90\right)$
$\hat{y}^{1}=5,42552+0,03804^{1} \mathrm{R}-0,09163^{1} \mathrm{D}-0,00048^{1} \mathrm{RD}+$ $0,00069^{1} \mathrm{D}^{2}\left(\mathrm{R}^{2}=0,69\right)$

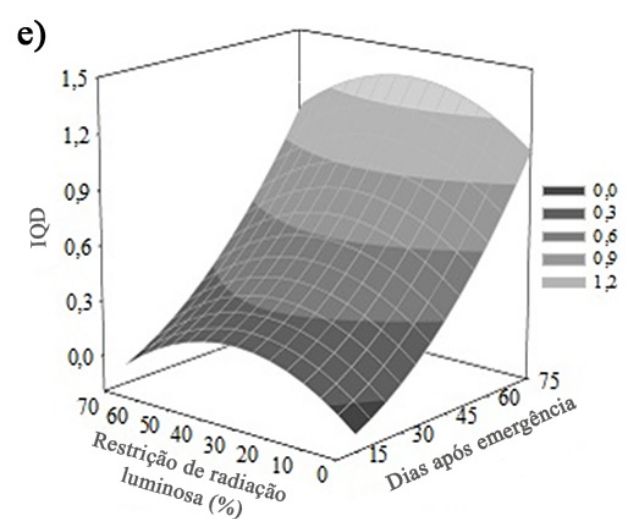

$$
\hat{y}^{1}=-0,18737+0,01606^{1} R+0,002251^{\text {ns }} D-0,00022^{1} R^{2}+0,00019^{1} D^{2}\left(R^{2}=0,81\right)
$$

FIGURA 2: Superfície de resposta em função da massa seca da parte aérea (a), massa seca da raiz (b), massa seca total (c), relação massa seca da parte aérea (MSPA)/massa seca da raiz (MSR) (d) e índice de qualidade de Dickson (IQD) (e) de Pterogyne nitens Tull. em função dos níveis de restrição de radiação luminosa (RL) e dias após a emergência (DAE) em Vitória da Conquista, Bahia. ns, ${ }^{5},{ }^{1}$ coeficientes da equação não significativo e significativo a 5 e $1 \%$, respectivamente.

FIGURE2: Response surface of Pterogyne nitens Tull. aerial part dry weight (a), root dry weight (b), dry weight total (c), aerial part dry weight (MSPA)/root dry weight (MSR) (d) and quality index of Dickson (IQD) (e) growing at radiance restriction levels (RL) and days after emergence (DAE) in Vitória da Conquista, Bahia. ${ }^{\text {ns }},{ }^{5},{ }^{1}$ coefficients of the equation not significant and significant the 5 and $1 \%$ respectively. 
(2002). Os mesmos autores verificaram para Hymenaeacourbaril que o sombreamento foi fator negativo para os incrementos de MSR, o mesmo foi verificado por Melo et al. (2008) para Enterolobium contortisiliquum. Resultados semelhantes ao do presente estudo foram verificados por Alvarenga et al. (2003) estudando Croton urucurana, onde a MSR foi maior sob 0 e $30 \%$ de RL, comparadas com 50 e 70\%. Caron et al. (2010) avaliando Schizolobium parahyba não verificaram diferença para MSR sob 0, 30, 50 e 70\% de RL.

Para todo o período de tempo do experimento, a relação MSPA/MSR (Figura 2d) foi crescente com aumento da RL, atingindo valores máximos estimados 6,36 com $70 \%$ de RL, aos 15 DAE. Entretanto, embora o comportamento tenha sido homogêneo, o efeito de RL para tal relação foi mais intenso no início do ensaio, ocorrendo aos 15 DAE uma diferença entre o tratamento a pleno sol em relação a $70 \%$ de RL de 2,15 . Ao final do ensaio, aos 75 DAE, na relação MSPA/MSR houve elevação de 0,14 para o tratamento com $70 \%$ de RL, em comparação às plantas mantidas a pleno sol. Quando foi analisada a relação entre MSPA/MSR e DAE para o tratamento a pleno sol foi verificado inicialmente tendência de decréscimos com discreta elevação de valores a partir dos 67 até 70 DAE. Do gradiente entre 0 até $23 \%$ de RL, os decréscimos de MSPA/MSR se estenderam por períodos maiores que 67 dias até o estabelecimento da tendência exclusiva de redução de MSPA/MSR em função dos dias, mantida de 24 até $70 \%$ de RL.

Foi verificada atenuação do efeito da restrição de luz em estágios de desenvolvimento posteriores, observada por meio do decréscimo de valores da relação MSPA/MSR (4,21 e 6,36, para 0 e $70 \%$ de sombreamento aos 15 DAE 2,43 e 2,58 para 0 e $70 \%$ de sombreamento aos $75 \mathrm{DAE}$ ), bem como de sua variação durante o período do estudo (variação de 2,16 aos 15 DAE e 0,14 aos 75 DAE). Portanto, para a espécie em estudo, foi verificado que, para os estágios de desenvolvimento iniciais, houve elevada plasticidade morfológica, ocorrendo atenuação deste fenômeno nos estágios mais tardios. Segundo Birchler et al. (1998), esta relação expressa a qualidade das mudas e, para a maioria das espécies, o valor ideal deve manter-se inferior a 2. Para o presente estudo, não foi atingido tal patamar, sendo os menores valores observados ao final do ensaio.

Valor máximo de IQD $(1,35)$ foi atingido aos 75 DAE e com 36,5\% de RL (Figura 2e), refletindo a estabilidade do comportamento das plantas de Pterogyne nitens em relação ao tempo de avaliação. O IQD é utilizado e citado por vários autores como um parâmetro adequado para expressar a qualidade das mudas, pois define a robustez e o equilíbrio da distribuição da biomassa na muda, com a ponderação simultânea de muitas variáveis (FONSECA et al., 2002; BERNARDINO et al., 2005; MELO et al., 2008; AZEVEDO et al., 2010; CRUZ et al., 2011). Quanto maior o valor do quociente do IQD, melhor o padrão de qualidade das mudas (GOMES, 2001). Desta forma, verificou-se que aproximadamente $35 \%$ de RL sejam as condições ideais de sombreamento para produção de mudas de Pterogyne nitens.

\section{CONCLUSÕES}

A restrição de luz até $70 \%$ induziu a alterações morfológicas típicas de mecanismos de escape ao sombreamento como elevação da altura e área foliar individual e total.

A redução de acúmulo de massa da raiz está relacionada a custos da ativação de mecanismos de escape ao sombreamento.

A restrição de $36,5 \%$ de luz favorece a obtenção de mudas de Pterogyne nitens de elevada qualidade aos 75 dias após plantio.

\section{REFERÊNCIAS BIBLIOGRÁFICAS}

ALABADÍ, D., BLAZQUEZ, M. A. Integration of light and hormone signals. Plant Signaling and Behavior, Austin, v.3, n.7, p. 448-449, July 2008. ALVARENGA, A. A.et al.Effects of different light levels on the initial growth and photosynthesis of croton urucuranabaill. in southeastern Brazil. Revista Árvore, Viçosa, v. 27, n. 1, p. 53-57, jan./fev. 2003.

ANTEN, N. P. R. et al.Interactive Effects of Spectral Shading and Mechanical Stress on the Expression and Costs of Shade Avoidance.The American Naturalist,Chicago, v, 173, n.2, p.241-255, Feb. 2009.

AZEVEDO, I. M. G. et al.Estudo do crescimento e qualidade de mudas de marupá (Simarouba amara Aubl.) em viveiro.Acta Amazonica, Manaus, v. 40, n. 1, p. 157-164, jan./mar. 2010.

BERNARDINO,D.C.S.etal.Crescimentoequalidade de mudas de AnadenantheraMacrocarpa(Benth.) Brenan em resposta à saturação por bases do substrato. Revista Árvore, Viçosa, v. 29, n. 6, 
p. 863-870, nov./dez. 2005.

BIRCHLER, T.et al. La planta ideal: revisiondel concepto, parâmetros definitorios e implementactionpractica. Investigacion Agraria, Sistemas y Recursos Forestales, Madrid, v. 7, n.1/2, p.109-121,1998.

CAMPOS, M. A. A.; UCHIDA, T. Influência do sombreamento no crescimento de mudas de três espécies amazônicas. Pesquisa Agropecuária Brasileira, Brasília, v. 37, n. 3, p. 281-288, mar. 2002.

CARON, B. O. et al.Crescimento em viveiro de mudas de Schizolobiumparahyba(vell.) s. f. blake. submetidas a níveis de sombreamento.Ciência Florestal, Santa Maria, v. 20, n. 4, p. 683-689, out./dez. 2010.

CARVALHO, P. E. R. Espécies florestais brasileiras:recomendações silviculturais, potencialidades e uso da madeira. Colombo: EMBRAPA-CNPMF, 1994, 640 p.

CESAR, F. R. C. F.et al.Morfofisiologia foliar de cafeeiro sob diferentes níveis de restrição luminosa. Coffee Science, Lavras, v. 5, n. 3, p. 262-271, set./dez. 2010.

CHAVES,A.S.;PAIVA,H.N. Influênciadediferentes períodos de sombreamento sobre a qualidade de mudas de fedegoso (Senna macranthera(Collad.) Irwin et Barn.). Scientia Florestalis, Piracicaba, n. 65 , p. 22-29, jun. 2004.

CRUZ, C. A. F.et al.Crescimento e qualidade de mudas de Fedegoso cultivadas em latossolo vermelho-amarelo em resposta a macronutrientes. Scientia Florestalis, Piracicaba, v. 39, n. 89, p. 021-033, mar. 2011.

DICKSON, A.; LEAF, A.; HOSNER, J. F. Quality appraisal of white spruce and white pine seedling stock in nurseries. Forestry Chronicle, West Mattawa, v. 36, n. 1, p. 10-13, Jan./Feb. 1960.

FERREIRA, F. G. et al.Avaliação de mutagenicidade e antimutagenicidade de diferentes frações de Pterogynenitens(Leguminosae), utilizando ensaio de micronúcleo em Tradescantiapallida.Revista Brasileira de Farmacognosia, João Pessoa, v. 19, n. 1A, p. 61-67. jan./mar. 2009.

FILARDI, F. L. R. et al.Padrões de distribuição geográfica de espécies arbóreas de Leguminosae ocorrentes no cerrado.Revista Brasileira de Biociências, Porto Alegre, v. 5, supl. 2, p. 1116-1118, jul. 2007.

FILARDI, F. L. R., GARCIA, F. C. P., OKANO, R. M. C. Caesalpinoideae (Leguminosae) lenhosas na estação ambiental de Volta Grande, Minas
Gerais, Brasil. Revista Árvore, Viçosa, v. 33, n. 6, p. 1071-1084. nov./dez. 2009.

FONSECA, E. P.et al. Padrão de qualidade de mudas de Trema micrantha(L.) Blume, produzidas sob diferentes períodos de sombreamento. Revista Árvore, Viçosa, v. 26, n. 4, p. 515-523, jul./ago. 2002.

FRANCO, A. M. S., DILLENBURG, L. R. Ajustes morfológicos e fisiológicos em plantas jovens deAraucariaangustifolia (Bertol.) Kuntze em resposta ao sombreamento. Hoehnea, São Paulo, v. 34, n. 2, p. 135-144. abr.jun. 2007.

GRIME, J. P. Evidence for the existence of three primary srategies in plants and its relevance to ecological and evolutionary theory. The American Naturalist, Chicago, v. 982, n. 3, p. 1169-1194, Nov./Dec. 1977.

HALLIDAY, K., MARTINEZ-GARCIA, J. .F, JOSSE, E. Integration of light and auxin signaling. Cold Spring Harbor Perspectives Biology, New York, v. 1, n. 6, p. 1-11, Dec. 2009.

LAXMI, A. et al. Light plays an essential role in intracellular distribuition of auxin efflux carrier PIN2 in Arabidopsis thaliana. Plos One, San Francisco, v. 3, e1510, p.1-11. Jan.2008.

LEAL, P. L. et al.Crescimento de mudas micropropagadas de bananeira micorrizadas em diferentes recipientes.Revista Brasileira de Fruticultura, Jaboticabal, v. 27, n. 1, abr. 2005.

LORENZI, H. Árvores Brasileiras: manual de identificação e cultivo de plantas arbóreas nativas do Brasil.: Plantarum, Nova Odessa,v. 1, p.384, 2002.

LUSK, C. Leaf area and growth of juvenile temperate evergreens in low light: species of contrasting shade tolerance change rank during ontogeny. Functional Ecology, London, v. 18, n. 6, p. 820-828, Nov./Dec.2004.

LUSK, C. H. et al.Ontogenic variation in light requirements of juvenile rainforest evergreens.

Functional Ecology, London,v. 22, n. 3, p. 454-459, June2008.

MARTINS NETO, F. L.; OLIVEIRA JÚNIOR, O.; MATSUMOTO, S. N. Temperatura da água e tempo de exposição na superação da dormência de sementes de Pterogynenitens Tul. In: CONGRESSO BRASILEIRO DE AGROECOLOGIA, 6., 2009, Curitiba. Anais... Pinhais: Associação Brasileira de Agroecologia, Revista Brasileira de Agroecologia, 2009, v. 4, n. 2. p.1155-1158.

MELO, R. R. et al.Crescimento inicial de mudas de Enterolobiumcontortisiliquum(Vell.) Morong. 
sob diferentes níveis de luminosidade. Revista Brasileira de Ciências Agrárias, Recife, v. 3, n. 2, p. 138-144, abr.jun. 2008.

MORAIS NETO, S. P.et al. Crescimento de mudas de algumas espécies arbóreas que ocorrem na mata atlântica em função do nível de luminosidade. Revista Árvore, Viçosa, v. 24, n. 1, p. 35-45, jan./fev. 2000.

MORELLI, G.; RUBERTI, I. Shade avoidance responses: driving auxin along lateral routes. Plant Physiology, Rockville, v. 122, p. 621-626, March 2000.

NIINEMETS, U. Responses of forest trees to single and multiple environmental stresses from seedlings to mature plants: Past stress history, stress interactions, tolerance and acclimation. Forest Ecologyand Management, Amsterdam, v. 260, n. 10, p. 1623-1639, Oct. 2010.

PELLIZZARO, K.et al.Superação da dormência e influência do condicionamento osmótico em sementes de Pterogynenitenstul. (Fabaceae). Revista Caatinga, Mossoró, v. 24, n. 3, p. 1-9, jul./set. 2011.

POORTER, L., KITAJIMA, K. Carbohydrate storage and light requirementes of tropical moist and dry forestry tree species.Ecology, Washington, v. 88, n.4, p. 1000-1011,Apr. 2007.

REGAZINI, L. O. et al.Constituintes químicos das flores de Pterogyne nitens (Caesalpinioideae). Química Nova, São Paulo, v. 31, n.4, p. 802-806. 2008.

RESENDE, S. V.et al.Influência da luz e substrato na germinação e desenvolvimento inicial de duas espécies de CalliandraBenth. (Mimosoideae Leguminosae) endêmicas da Chapada Diamantina,
Bahia. Revista Árvore, Viçosa, v. 35, n. 1, p. 107-117, jan./fev. 2011.

RUBERTI, I.; SESSA, G. et al. Plant adaptation to dynamically changing environment: the shade avoidance response. Biotechnology Advances, New York, doi: 10.1016/j.biotechady.2011.08.014.2011.

SAS INSTITUTE. SAS for linear models: a guide to the ANOVA and GLM procedures. Cary, 1985.

STEPANOVA, A. N.et al. Taa1-mediated auxin biosynthesis is essential for hormone crosstalk and plant development. Cell, Massachusetts, v. 133, n. 1, p.177-191, Apr. 2008.

TAO, Y. et al.Rapid synthesis of auxin via a new tryptophan-dependent pathway is required for shade avoidance in plants. Cell, Massachusetts, v. 133, n. 1, p. 164-76, Apr. 2008.

TEIXEIRA, P. T. L.et al.Desenvolvimento vegetativo de porta-enxertos de citros produzidos em diferentes recipientes. Ciência Rural, Santa Maria, v. 39, n.6, p. 1695-1700, jun. 2009.

VALLADARES, F.; NIINEMETS, Ü. Shade tolerance, a key plant feature of complex nature and consequences. Annual Review of Ecology and Evolution Systems, Palo Alto, v. 39, p.237 - 257. 2008.

VALLADARES, F., SALDAÑA, A., GIANOLI, E. Costs versus risks: Architectural changes with changing light quantity and quality in saplings of temperate rainforest trees of different shade tolerance. Austral Ecology, Richmond,doi: 10.1111/ j.1442-9993.2011.02245.2011.

VEGLIO, A. The shade avoidance syndrome: a non-Markovian stochastic growth model. Journal of Theoretical Biology, London, n. 264, n.3, p. 722-728, June 2010. 